\title{
Kohlenmonoxid-Intoxikationen Hyperbare Sauerstofftherapie kann neurologische Spätschäden vermeiden
}

\author{
A. H. Leischker, Oberarzt Abteilung Innere Medizin, St. Bonifatius Hospital, Lingen/Ems
}

NOTFALLMEDIZIN 2003; 29: 88-90

\begin{abstract}
Die präklinische Diagnose einer Kohlenmonoxidvergiftung kann schwierig sein - Anamnese und Beobachtung der Umgebungsbedingungen sind jedoch oft wegweisend. Da es sich um ein hochtoxisches und explosives, farb- und geruchloses Gas handelt, muss auf einen entsprechenden Eigenschutz unbedingt geachtet werden. Wenn der Verdacht besteht, dass in geschlossenen Räumen Kohlenmonoxid vorhanden ist, sollte immer die Feuerwehr mitoder nachalarmiert werden. Nur sie verfügt über den erforderlichen „schweren Atemschutz“ (Pressluftatemgeräte) und die entsprechenden Gasspürgeräte.

Präklinisch sollten die betroffenen Patienten umgehend Sauerstoff in möglichst hoher Konzentration erhalten. Die Indikation zur Intubation und Beatmung (mit $\left.100 \% \mathrm{O}_{2}\right)$ ist großzügig zu stellen. Akutkomplikationen, wie zerebrale Krampfanfälle oder Herzrhythmusstörungen, werden symptomatisch behandelt. In einer neueren Studie konnte nun eindeutig nachgewiesen werden, dass eine frühzeitig eingeleitete hyperbare Sauerstofftherapie in einer Druckkammer neurologische Spätschäden verhindern kann. Patienten mit schwerer Kohlenmonoxidintoxikation sollten deshalb nach Möglichkeit in ein Krankenhaus mit angeschlossenem Druckkammerzentrum transportiert werden.
\end{abstract}

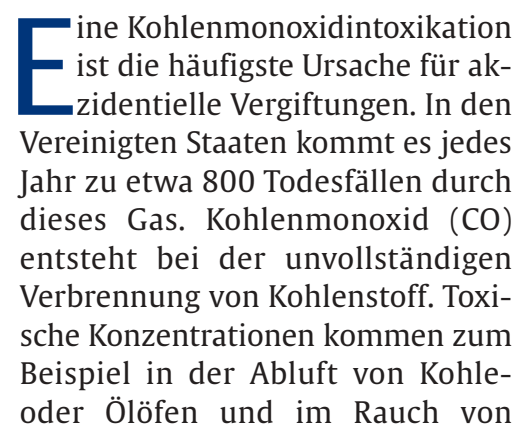

Schwelbränden vor. Autoabgase enthalten bis zu 20\% Kohlenmonoxid. Tödliche Folgen sind bereits bei einer Kohlenmonoxidkonzentration von mehr als $0,2 \%$ in der Umgebungsluft zu erwarten.

\section{Heimtückisches Gas}

Bei normaler Umgebungstemperatur ist Kohlenmonoxid ein stabiles Gas, welches schnell durch die Ka-

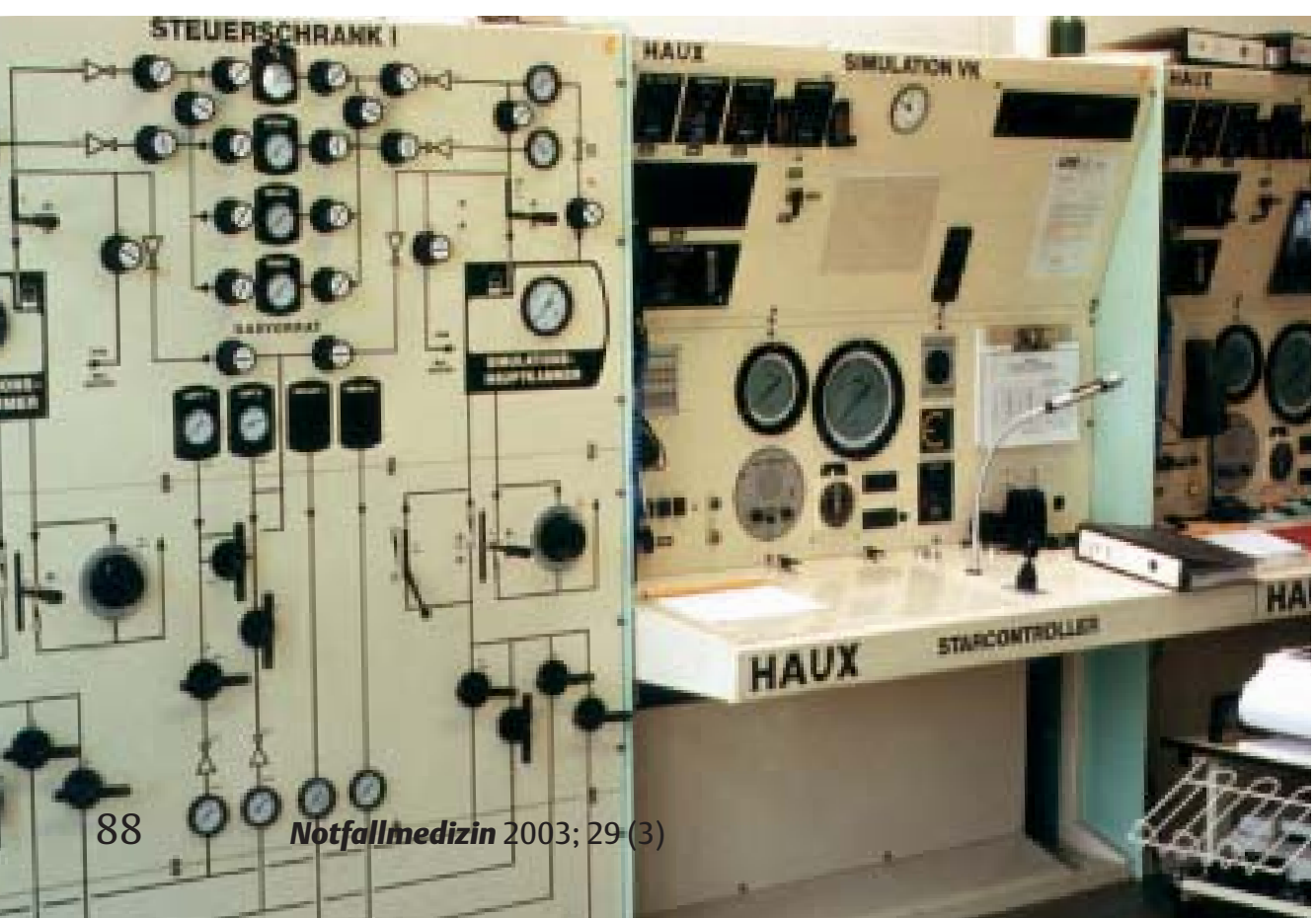

pillaren der Alveolen diffundiert (Tab. 1). Claude Bernard hat 1865 erstmalig herausgefunden, dass die toxische Wirkung von Kohlenmonoxid durch die Bildung von Carboxyhämoglobin ( $\mathrm{CO}-\mathrm{Hb})$ bedingt ist. Kohlenmonoxid hat eine 300mal höhere Affinität zu Hämoglobin als Sauerstoff. Dadurch wird der Sauerstoff aus seiner Bindung mit dem roten Blutfarbstoff verdrängt. Carboxyhämoglobin verringert den Sauerstoffgehalt des Blutes und verhindert, dass der an Hämoglobin gebundene Sauerstoff an das Gewebe abgegeben werden kann. Nachdem Kohlenmonoxid in die Körperzelle aufgenommen wurde, entfaltet es seine toxische Wirkung auch über von der Hypoxämie unabhängige Wirkungen, wie z.B. die Bindung an mitochondriale Cytochromoxidasen.

\section{Unspezifische Symptomatik}

Die Symptomatik einer Kohlenmonoxidvergiftung ist meist unspezifisch: die häufigsten Symptome sind Kopfschmerzen, Schwindel und Verwirrtheit (Tab. 2). Wenn eine Expositionsquelle nicht eindeutig zu erkennen ist, kann die Diagnosestellung - insbesondere bei leichteren Vergiftungen - schwierig sein. Man geht davon aus, dass mehr als $5 \%$ aller Patienten, welche sich in den Wintermonaten in einer Notfallambulanz vorstellen, an einer - meist unerkannten - Kohlenmonoxidvergiftung leiden.

Durch endogene Produktion von Kohlenmonoxid und die Aufnahme geringer Mengen von CO aus der normalen Umgebungsluft, liegt der Carboxyhämoglobinspiegel beim Gesunden normalerweise zwischen 1 und 2\%. Raucher haben einen deutlich erhöhten $\mathrm{CO}-\mathrm{Hb}$ Spiegel. 


\section{Tab. 1 Eigenschaften von Kohlenmonoxid}

- Chemische Formel: CO

- Farb-, geruch- und geschmacklos

- Hochentzündlich

- Gas-Luft-Gemisch ist hochexplosiv

Wer täglich ein Päckchen Zigaretten raucht, hat einen um etwa 5\% höheren Carboxyhämoglobinspiegel. Starke Raucher tolerieren - wenn sie keine Begleiterkrankungen haben Carboxyhämoglobinspiegel von bis zu 10\% ohne Symptome. Intoxikationszeichen treten normalerweise bei CO-Hb-Spiegeln von mehr als 15 bis $20 \%$ auf. Carboxyhämoglobinspiegel von über $25 \%$ gehen mit schweren Vergiftungserscheinungen einher und können zu plötzlicher Bewusstlosigkeit führen.

\section{Schwierige präklinische Diagnosestellung}

Das „klassische“, in den meisten notfallmedizinischen Lehrbüchern aufgeführte Symptom, die kirschrote Hautfarbe, ist fast nie anzutreffen. In der Tat haben $42 \%$ eine normale Hautfarbe, $40 \%$ sind blass-fahl und $18 \%$ zyanotisch.

Aufgrund der Ähnlichkeit des Infrarotspektrums von $\mathrm{CO}-\mathrm{Hb} \mathrm{zu}$ $\mathrm{O}_{2}-\mathrm{Hb}$ (Oxyhämoglobin) wird $\mathrm{CO}-\mathrm{Hb}$ bei der Pulsoxymetrie fälschlicherweise als $\mathrm{O}_{2}-\mathrm{Hb}$ interpretiert. Bei einer schweren Kohlenmonoxidintoxikation zeigt das Pulsoximeter deshalb eine Sauerstoffsättigung von über $98 \%$ an - obwohl der Patient tatsächlich unter einer massiven Hypoxie leidet!

Der CO-Gehalt der Umgebungsluft kann mit einem Gasspürgerät (z.B. Dräger) nachgewiesen werden. Dieses steht in den ABC-Zügen der meisten Feuerwehren zur Verfügung.

Die laborchemische Bestimmung des Carboxyhämoglobinspiegels ist präklinisch nicht möglich. Daher ist das Rettungsdienstpersonal in der täglichen Praxis bei der Diagnosestellung auf eine genaue Beobachtung der Umgebungsbedingungen und - wenn möglich - die Anamnese angewiesen.

\section{Komplikationen und Spätschäden}

Bei etwa 50\% aller Intoxikationen kommt es zu schwerwiegenden Komplikationen. Diese können in zwei Kategorien eingeteilt werden: Akute kardiale und neurologische Komplikationen sowie Spätschäden.

An akuten kardialen Komplikationen können tachykarde und bradykarde Herzrhythmusstörungen sowie eine myokardiale Ischämie auftreten. Akute neurologische Komplikationen manifestieren sich meist in Form zerebraler Krampfanfälle.

Das verzögert auftretende Neurologische Syndrom tritt bei etwa 15\% der Patienten mit schwerer Intoxikation nach einem Intervall von zwei bis 28 Tagen auf und äußert sich in Gedächtnisstörungen und anderen kognitiven Störungen. Bei 25 bis 50\% der Patienten, die im Rahmen der Intoxikation bewusstlos waren oder einen Carboxyhämoglobinspiegel von mehr als 25\% aufwiesen, treten kognitive Störungen auf, die länger als einen Monat anhalten.

\section{- Eigenschutz beachten}

Da Kohlenmonoxid hochtoxisch, geruch- und farblos sowie hochexplosiv ist, sollte grundsätzlich die Feuerwehr mitalarmiert oder nachgefordert werden. Die Rettung aus geschlossenen Räumen sollte nur mit schwerem Atemschutz (über diesen verfügt in der Regel nur die Feuerwehr) erfolgen. Die Scheiben geschlossener Räume (z.B. PKW, Häuser) sollten - wenn möglich eingeschlagen werden. Wegen der
Explosionsgefahr sollte weder offenes Licht verwendet noch elektrische Anlagen (z.B. Haustürklingel!) betätigt werden. Die Feuerwehr verfügt über explosionsgeschützte Handleuchten.

Selbstverständlich darf in einem mit Kohlenmonoxid gefüllten geschlossenen Raum keine Defibrillation durchgeführt werden.

\section{Basismaßnahmen}

Wie bei jedem Notfall sollten auch bei einem Patienten mit Kohlenmonoxidintoxikation zunächst die Vitalfunktionen überprüft und gegebenenfalls gesichert werden.

Bei Suizidversuchen (z.B. Einleiten von Autoabgasen in einen PKWInnenraum) muss immer auch an Mischintoxikationen (Alkohol, Medikamente, Pestizide) gedacht werden.

Sobald möglich, sollte Sauerstoff in möglichst hoher Konzentration appliziert werden. Sauerstoff bindet kompetitiv an Hämoglobin, wodurch die Halbwertszeit von $\mathrm{CO}-\mathrm{Hb}$ verkürzt wird. Bei einer $\mathrm{FIO}_{2}$ (inspiratorische $\mathrm{O}_{2}$-Konzentration) von $21 \%$ (entspricht Raumluft) beträgt die Halbwertszeit von Co-Hb vier bis sechs Stunden, bei einer $\mathrm{FIO}_{2}$ von $100 \% 40$ bis 60 Minuten und unter hyperbarer Sauerstofftherapie nur 15 bis 30 Minuten. Ideal ist die Applikation mittels Demand-Ventil, hierbei wird eine Konzentration von nahezu 100\% Sauerstoff erreicht. Die Indikation zur Intubation und Beatmung sollte großzügig gestellt werden. Bei kontrolliert beatmeten

\section{Tab. 2 Symptomatik der Kohlenmonoxidvergiftung}

CO-Hb-Gehalt im Blut Klinische Symptomatik

\begin{tabular}{ll}
\hline $1-3 \%$ & Normalwert bei Gesunden - keine Symptomatik \\
\hline$>5 \%$ & Visusbeeinträchtigung - bei starken Rauchern keine Symptomatik \\
\hline$>10 \%$ & Kopfschmerzen, Schwindel \\
\hline$>15 \%$ & Ohrensausen, Sehstörungen, Kurzatmigkeit, Tachykardie \\
\hline $20 \%$ & $\begin{array}{l}\text { Schwere Vergiftungszeichen. Zunehmende ZNS-Symptome: } \\
\text { Mattigkeit, teilweise auch Euphorie }\end{array}$ \\
\hline$>30 \%$ & Somnolenz \\
\hline$>40 \%$ & Bewusstlosigkeit und Krampfanfälle \\
\hline$>60 \%$ & $\begin{array}{l}\text { Koma, Hypoventilation bis zur zentralen Atemlähmung, } \\
\text { Metabolische Azidose }\end{array}$ \\
\hline$>70 \%$ & Tod innerhalb von 10 Minuten bis 1 Stunde \\
\hline
\end{tabular}

Neben der Höhe des CO-Hb spielt auch die Expositionsdauer eine wichtige Rolle. 


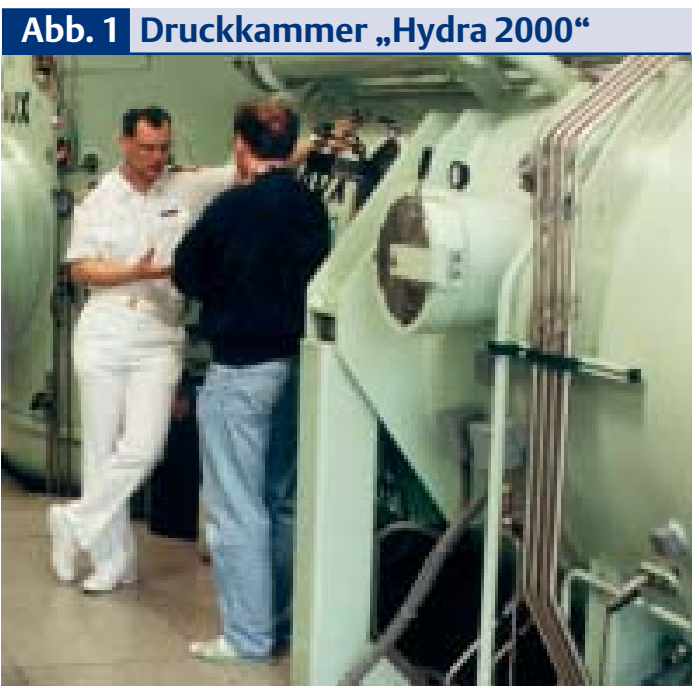

Druckkammer des Schifffahrtmedizinischen Instituts der Marine Kronshagen

Patienten sollte eine leichte Hyperventilation $\left(\mathrm{CO}_{2} 25-30 \mathrm{mmHg}\right)$ angestrebt werden.

Zerebrale Krampfanfälle können mit Diazepam therapiert werden. Die Hirnödemprophylaxe mit Glucocorticoiden ist umstritten und sollte deshalb nicht durchgeführt werden. Wegen der Gefahr von Herzrhythmusstörungen sollte ein kontinuierliches EKG-Monitoring erfolgen. Die Messung der Sauerstoffsättigung ergibt bei der Kohlenmonoxidintoxikation falsch hohe Werte und kann deshalb eine trügerische Sicherheit vermitteln. Neben der sorgfältigen klinischen Beobachtung sollte deshalb zur Überwachung der Beatmung - wenn verfügbar - die endexspiratorische $\mathrm{CO}_{2}$-Konzentration gemessen werden. Der Transport sollte grundsätzlich in Begleitung eines Notarztes erfolgen.

\section{Therapie in der Klinik}

In der Klinik wird zunächst die Therapie mit Applikation einer möglichst hohen Sauerstoffkonzentration fortgesetzt werden. Die Bestimmung des Carboxyhämoglobinspiegels ist in der Regel nur größeren Laborabteilungen möglich. Oft müssen deshalb auch im Krankenhaus die ersten Therapieentscheidungen nach der klinischen Symptomatik getroffen werden.

Lindell K. Weaver und Kollegen haben erstmals in einer großen randomisierten Doppelblindstudie an insgesamt 152 Patienten nachgewiesen, dass eine hyperbare Sauerstofftherapie neurologische Spätschäden bei Kohlenmonoxidintoxikation vermeiden kann, wenn damit innerhalb von 24 Stunden begonnen wird. Die Patienten wurden im Rahmen der Studie in der Druckkammer zunächst bei einem Luftdruck von 3 Atmosphären (304 kPa), dann bei einem Luftdruck von 2 Atmosphären (203 $\mathrm{kPa}$ ) mit $100 \%$ Sauerstoff behandelt (Abb. 1). Insgesamt wurden drei Druckkammersitzungen durchgeführt. Nach sechs Wochen hatten von den in der Druckkammer behandelten Patienten nur 25\% kognitive Störungen. Dagegen litten von den mit normobarem Sauerstoff (ohne Druckkammer) behandelten Patienten $46 \%$ an kognitiven Störungen. Auch zwölf Monate nach der Intoxikation hatten die mit hyperbarem Sauerstoff (in der Druckammer) behandelten Patienten deutlich seltener kognitive Einschränkungen als die mit 100\% Sauerstoff unter normobaren Bedingungen therapierten Patienten. Wegen der eindrücklichen Vorteile der Druckkammerbehandlung wurde die Studie vorzeitig abgebrochen und alle folgenden Patienten der hyperbaren Therapie zugeführt.

Es konnte somit eindeutig nachgewiesen werden, dass eine innerhalb von 24 Stunden nach Kohlenmonoxidintoxikation eingeleitete Druckkammerbehandlung neurologische Spätschäden verhindern kann. Bei schweren Kohlenmonoxidvergiftungen sollte die hyperbare Sauerstofftherapie deshalb Standard sein. Vermutlich ist die Druckkammerbehandlung umso effektiver, je früher damit begonnen wird (möglichst innerhalb von sechs Stunden).

Patienten mit schwerer Kohlenmonoxidintoxikation sollten deshalb - wenn möglich - in ein Krankenhaus transportiert werden, welches über eine Druckkammer mit 24-StundenBereitschaft verfügt. Die Rettungsleitstellen verfügen über entsprechende Listen von Druckkammerzentren und können vorab klären, ob die jeweilige Druckkammer einsatzbereit ist. Eine Voranmeldung sollte grundsätzlich erfolgen, da bei manchen Druckkammerzentren Techniker und Ärzte mit spezieller Erfah- rung in Überdruckmedizin außerhalb der regulären Dienstzeiten im Rahmen ihres Rufbereitschaftsdienstes frühzeitig alarmiert werden müssen.

\section{Summary}

Although the out-of-hospital diagnosis of carbon monoxide poisoning may be difficult, the history and observation of ambient conditions are often indicative. Since we are here dealing with a highly toxic and explosive, colourless and odourless gas, the physician must take appropriate self-protective measures. Whenever carbon monoxide is suspected to have accumulated within a closed space, the fire department should be called in, since it, alone, is in possession of the necessary breathing equipment (compressed air respirators) and appropriate gasdetecting devices. In the out-ofhospital setting, the victim should immediately be given oxygen at as high a concentration as possible. The indication for intubation and ventilation (with $100 \%$ oxygen) must be established liberally. Such acute complications as cerebral convulsions or cardiac arrhythmias receive symptomatic treatment. A more recent study has unequivocally demonstrated that early institution of hyper baric oxygen treatment in a compression chamber can prevent neurological sequelae. For this reason, patients suffering severe carbon monoxide poisoning should wherever possible transferred to a hospital with access to a facility offering such treatment.

\section{Literatur}

1. Weaver LK, Hopkins RO, Chan KJ et al. Hyperbaric Oxygen for Acute Carbon Monoxide Poisoning. N Engl J Med 2002; 347: 1057-1067

2. Thom SR. Hyperbaric-Oxygen Therapy for Acute Carbon Monoxide Poisoning (Editorial). N Engl J Med 2002; 347: 1105-1106

3. Piantadosi CA. Carbon Monoxide Poisoning (Perspective). N Engl J Med 2002; 347: 1054-1055

\section{Anschrift des Verfassers}

Dr. med. Andreas H. Leischker Facharzt für Innere Medizin, Rettungsmedizin, Tauchmedizin (GTÜM e.V.) St. Bonifatius Hospital

Oberarzt Abteilung Innere Medizin Wilhelmstraße 13, 49808 Lingen (Ems) Fax: 05 91/910-12 91

E-Mail: andreas.leischker@bonifatius-lingen.de 